\title{
EDITORIAL AND \\ PRODUCTION STAFF
}

Random House First Edition

EXECUTIVE EDITOR

Sol Steinmetz

DEVELOPMENTAL EDITOR

Marjorie McShane

SUPERVISING COPY EDITOR

Judy Kaplan

ENGLISH TEXT EDITOR

Joyce O'Connor

Linguistic CONSULTANTS

Jurij Apresjan

Igor Mel'čuk

Production Director

Patricia Ehresmann

BIBLIOGRAPHY

Kay L. Shaffer

INDEX PROGRAM

Boris Yamrom
Yale University Press Revised Edition

SPONSORING EDITOR

Vadim Staklo

Director of Publishing Operations

Christina H. Coffin

EDITOR, World LANGUAGES

Tim Shea

ENGLISH TEXT EDITOR

Judith Hehir

COPY EDITOR

Karen Hohner

PRODUCTION EDITOR

Ann-Marie Imbornoni

TECHNICAL SUPPORT AND COMPOSITION Kevin Krugh, Technologies 'N Typography

INDEX PROGRAM

Boris Yamrom 